\title{
Lista das espécies de Ropalomeridae, Sphaeroceridae e Ulidiidae (Diptera, Acalyptratae) do estado de Mato Grosso do Sul, Brasil
}

\author{
Karla Pessôa Tepedino', Anna Carolina Prestes' ', Cecília Kosmann' ', Ana Carolina Franco², \\ José Roberto Pujol-Luz' \& Cristiane V. A. Pujol-Luz²
}

\begin{abstract}
1. Universidade de Brasília, Instituto de Ciências Biológicas, Departamento de Zoologia, 70910-900 Brasília, DF. (karlaptp@gmail.com) 2. Universidade Católica de Brasília, Laboratório de Zoologia, QS 7 lote 1, BI. M s.331, 72030-170 Taguatinga, DF.
\end{abstract}

Recebido 8 dezembro 2016

Aceito 6 fevereiro 2017

DOI: $10.1590 / 1678-4766 e 2017147$

ABSTRACT. Checklist of species of Ropalomeridae, Sphaeroceridae and Ulidiidae (Diptera, Acalyptratae) of the state of Mato Grosso do Sul, Brazil. In this paper it is provided a checklist of the Ropalomeridae, Sphaeroceridae and Ulidiidae species registered in the state of Mato Grosso do Sul, Brazil. The Ropalomeridae is represented by eight species distributed in four genera: Dactylissa Fischer (1), Kroeberia Lindner (1), Ropalomera Wiedemann (5) and Willistoniella Mik (1). A single species of Sphaeroceridae is recorded for the state: Neosphaerocera flavicoxa (Malloch, 1925), as well as of Ulidiidae: Notogramma cimiciforme Loew, 1868.

KEYWORDS. Biodiversity, Cerrado, Pantanal, Biota-MS Program.

RESUMO. Neste trabalho é apresentada uma lista de espécies de Ropalomeridae, Sphaeroceridae e Ulidiidae registradas no estado do Mato Grosso do Sul, Brasil. Ropalomeridae é representada por oito espécies distribuídas em quatro gêneros: Dactylissa Fischer (1), Kroeberia Lindner (1), Ropalomera Wiedemann (5) e Willistoniella Mik (1). Apenas uma espécie de Sphaeroceridae possui registro para o estado: Neosphaerocera flavicoxa (Malloch, 1925), assim como de Ulidiidae: Notogramma cimiciforme Loew, 1868.

PALAVRAS-CHAVE. Biodiversidade, Cerrado, Pantanal, Programa Biota-MS

Os Ropalomeridae são dípteros com distribuição predominantemente Neotropical e uma única espécie conhecida na Região Neártica (MARQuES \& AlE-RochA, 2005). Estão classificados dentro da superfamília Sciomyzoidea, formando um agrupamento com Coelopidae, Dryomyzidae, Helosciomyzidae, Sciomyzidae e Sepsidae (IBÁÑEZ-BERNAL \& HERNÁNDEZ-OrTIZ, 2010). Atualmente a família inclui 26 espécies distribuídas entre oito gêneros. No Brasil são conhecidas 24 espécies em seis gêneros (MARQUES \& Ale-Rocha, 2005).

Os ropalomerídeos são facilmente reconhecidos por possuírem o vértex côncavo, face com protuberância mediana, ponte pré-coxal presente, grande ampola desenvolvida, veia $M$ dobrada para frente, tíbia posterior lateralmente comprimida e fortemente expandida, entre outros (MCALPINE, 1981; IbáÑeZ-Bernal \& HernándeZ-Ortiz, 2010). Pouco se conhece sobre a biologia das espécies da família. LOPES (1932) descreveu o ovo e a larva de Ropalomera stictica Wiedemann, 1830 e ilustrou o pupário de Ropalomera clavipes (Fabricius, 1805). FISCHER (1932) descreveu o pupário de Willistoniella pleuropunctata (Wiedemann, 1824). Posteriormente, Prado (1966) observou os ovos destas espécies (IbÁÑEZ-BERnAL \& HernándeZ-OrTIZ, 2010).

Os Sphaeroceridae (Acalyptratae, Sphaeroceroidea) são dípteros cosmopolitas de pequeno porte $(0,7 \mathrm{a} 6 \mathrm{~mm}) \mathrm{com}$ a coloração variando do marrom ao preto. São facilmente reconhecidos pela redução do primeiro tarsômero da perna posterior (MARSHALl \& RichaRdS, 1987; MARSHALL \& BUCK, 2010). Mundialmente foram descritas mais de 1.571 espécies, com 350 delas conhecidas para a Região Neotropical. Destas, 70 ocorrem no Brasil (RoHÁČEK et al., 2001; BERGERON, 2010; Marshall et al., 2011; PAPE et al., 2011).

São moscas que habitam locais úmidos e estão associadas com a decomposição de matéria orgânica, alimentando-se de microorganismos presentes nestes substratos (MARshall \& BucK, 2010). Dentre os recursos explorados citam-se fezes (Mendes \& Linhares, 2002), material vegetal em decomposição e fungos (Buck, 1997), bancos de algas marinhas (BELSHAW, 1989), matéria orgânica em cavernas (BARNES et al. 2009) e carcaças de vertebrados e invertebrados (Norrbom \& KIM, 1984; BucK, 1997). Além desses, algumas espécies de Sphaeroceridae são foréticos de artrópodes como besouros (NIOGRET \& LUMARET, 2009), caranguejos (GóMEZ, 1977) e formigas (RoHÁČEK \& Marshall, 1998).

Não apresentam importância econômica significativa (RoHÁČEK et al., 2001) embora possam gerar danos ao cultivo de fungos (Buxton, 1960; Marshall \& Richards, 1987). 
Sua importância médica é também pequena, mas há registros de Poecilosomella angulata (Thomson, 1869) causando miíase intestinal em humanos (Micks \& McKibBEN, 1956), além da transmissão de Salmonella sp. (MANRIQUE-SAIDE \& Delfín-GonZÁLeZ, 1997).

Os Ulidiidae (=Otitidae) (Acalyptratae, Tephritoidea) são dípteros predominantemente neotropicais, pequenos a médios (2 a $14 \mathrm{~mm}$ ), com a coloração amarela ao preta, podendo apresentar iridescência azul ou verde e asas geralmente manchadas (KAMENEVA \& KORNEYEV, 2010). É a terceira família com maior número de espécies dentro de Tephritoidea, apresentando 678 espécies no Mundo (Kameneva \& Korneyev, 2010; PAPE et al., 2011), das quais pelo menos 285 estão presentes na Região Neotropical e cerca de 60 espécies ocorrem no Brasil (STEYSKal, 1968).

Alguns adultos desta família são polinizadores (Ribeiro et al., 2006), mas geralmente são atraídos por matéria orgânica vegetal em decomposição como troncos, folhas, frutos (Teskey, 1976; Allen \& Foote, 1992), carcaças (CornabY, 1974; SouZa \& Linhares, 1997), fezes (MARChiori \& SiLva, 2001), dentre outros. Algumas espécies apresentam larvas fitófagas e se comportam como pragas agrícolas de cultivares como milho (DiAz, 1982; BertolacCini et al., 2010; Goyal et al., 2010), agave (BRUNEl \& Rull, 2010), maracujá (AguiAR-Menezes et al., 2004) e beterraba (BJERKe et al., 1992). Além dos danos causados pelas próprias larvas, a atividade destas facilita a entrada de patógenos nas plantas, podendo ocasionar perdas de até 100\% da produção (DiAz, 1982; BJERKE et al., 1992). Também carreiam bactérias entéricas, sendo responsáveis pela transmissão de infecções (BARNABE et al., 2007).

\section{MATERIAL E MÉTODOS}

A lista de espécies de Ropalomeridae registradas para o estado do Mato Grosso do Sul foi elaborada com base principalmente nos registros do catálogo da Região Neotropical de STEYSKal (1967) e nos trabalhos de STEYSKal (1957), Prado (1963), Marques \& Ale-Rocha (2005) e Boff et al. (2008).

A lista de espécies de Sphaeroceridae registradas para o Mato Grosso do Sul foi elaborada com base nos registros do catálogos de RichARDS (1967) para a Região Neotropical, RoHÁČEK et al. (2001) para o Mundo e a complementação deste último (MARSHALl et al., 2011). Bibliografia adicional do grupo também foi consultada: Richards (1965), Kim (1972), Mourgués-SCHURTer (1981), RoháČEK \& Marshall (1988, 1998), Marshall \& Norrbom (1992), Marshall (1995, 1997a,b, 2001), SMith \& Marshall (2004), Marshall et al. (2007), BuCK \& Marshall (2009) e BERGERON (2010).

A lista de espécies de Ulidiidae registradas para o estado do Mato Grosso do Sul foi elaborada com base nos registros do catálogo para a Região Neotropical de STEYSKAL (1968). Para a complementação da informação, a seguinte bibliografia adicional do grupo também foi avaliada: UcHôAFernandes et al. (2002, 2003), Aguiar-Menezes et al. (2004), Ribeiro et al. (2006), Rodrigues et al. (2006), BARNABE et al. (2007) e CRUZ et al. (2011).

\section{RESULTADOS E DISCUSSÃO}

Existem registros de ocorrência de seis gêneros e 24 espécies de ropalomerídeos no Brasil, dos quais quatro gêneros e oito espécies ocorrem no estado do Mato Grosso do Sul. Esta diferença no número de espécies registradas deve-se, provavelmente, à escassez de estudos envolvendo esta família de dípteros, uma vez que ela não possui interesse econômico aparente.

Apenas uma espécie de Sphaeroceridae foi registrada no estado do Mato Grosso do Sul: Neosphaerocera flavicoxa (Malloch, 1925). Este número certamente não representa a diversidade de espécies de esferocerídeos nesta região do Brasil. Outras sete espécies são conhecidas na Região Centro-Oeste, sendo seis dessas no estado do Mato Grosso (Richards, 1967; BucK \& Marshall, 2009; Bergeron, 2010). As espécies restantes relatadas no Brasil ocorrem em estados com maior tradição em pesquisas entomológicas como, por exemplo, São Paulo, Rio de Janeiro, Paraná, Santa Catarina e Pará (RohÁčé et al., 2001; MARShall et al., 2011). Para o melhor conhecimento da diversidade dos Sphaeroceridae no Mato Grosso do Sul, estudos mais aprofundados são necessários, incluindo coletas direcionadas para este grupo de moscas, levando-se em consideração a biologia e métodos de coleta adequados (BUCK, 1997). O conhecimento dos Sphaeroceridae da Região Neotropical é igualmente incipiente, havendo muitas espécies a serem descritas (Marshall, 1995; Marshall et al., 2007; Marshall \& BuCK, 2010), sendo possível afirmar que o baixo número de espécies registrado no Mato Grosso do Sul é decorrente do pouco conhecimento da dipterofauna da região.

Foi registrada no estado do Mato Grosso do Sul uma espécie da família Ulidiidae: Notogramma cimiciforme Loew, 1868 (UCHÔA-FERNANDES et al., 2002). O mesmo estudo cita a coleta de outras espécies de Ulidiidae, sem identificá-las. Além dessas, há uma espécie não identificada do gênero Euxesta Loew na região Centro-Oeste. O baixo número de espécies encontradas se deve à falta de especialistas no País, impossibilitando a identificação de espécimes coletados (UchôA-FERNANDES et al., 2003; RoDRIGUES et al., 2006); além da taxonomia pouco resolvida do grupo, com muitos gêneros carecendo de revisão (Foote, 1960; KAMENEVA \& KoRNEYEV, 2006, 2010). As principais revisões realizadas nas últimas décadas são da fauna da América Central (StEYSKal, 1971; KAMENEva, 2004a,b, 2005, 2009) e os principais trabalhos realizados no Brasil são relativos às poucas espécies de importância econômica (CRUZ et al., 2011), sendo essa uma amostragem defasada da fauna local se considerarmos a biologia do grupo. 
Lista de espécies de Ropalomeridae no Estado do Mato Grosso do Sul

\section{Dactylissa Fischer, 1932}

digiticornis Fischer, 1932. Localidade-tipo: Brasil, Mato Grosso (Mato Grosso do Sul), Fazenda Murtinho. Distribuição Neotropical: Brasil [Guanabara (Rio de Janeiro), Mato Grosso (Mato Grosso do Sul)], Paraguai (Villarica).

\section{Kroeberia Lindner, 1930}

chryserea Prado, 1966. Localidade-tipo: Brasil, Mato Grosso (Mato Grosso do Sul), Salobra, macho; São Paulo, Araçatuba, fêmea. Distribuição Neotropical: Brasil.

\section{Ropalomera Wiedemann, 1824}

clavipes (Fabricius, 1805). Localidade-tipo: América do Sul. Distribuição Neotropical: Guiana, Suriname, Equador, Brasil, Bolívia, Paraguai.

distincta Prado, 1966. Localidade-tipo: Brasil, Mato Grosso

(Mato Grosso do Sul), Fazenda Murtinho. Distribuição Neotropical: Brasil.

femorata (Fabricius, 1805). Localidade-tipo: América do

Sul. Distribuição Neotropical: sul do Brasil, México

(Yucatán), Bolívia, Paraguai e Argentina (Misiones).

glabrata Prado, 1966. Localidade-tipo: Brasil, Mato Grosso

(Mato Grosso do Sul), Três Lagoas, Fazenda Floresta.

Distribuição Neotropical: Brasil.

guimaraesi Prado, 1966. Localidade-tipo: Brasil, Mato

Grosso (Mato Grosso do Sul), Três Lagoas, Fazenda

Floresta. Distribuição Neotropical: Brasil.

\section{Willistoniella Mik, 1895}

pleuropunctata (Wiedemann, 1824). Localidade-tipo: "América do Sul". Distribuição Neotropical: Brasil, Mato Grosso (Mato Grosso do Sul, Três Lagoas, Fazenda Floresta), sul da América do Sul ao norte da Argentina, sul do México ao Panamá, Trindade.

\section{Lista de espécies de Sphaeroceridae no Estado do Mato Grosso do Sul}

\section{Neosphaerocera Kim, 1972}

flavicoxa (Malloch, 1925). Localidade-tipo: Costa Rica, San Mateo, Higuito. Distribuição Neotropical: Belize, Brasil, Colômbia, Costa Rica, El Salvador, Equador, Guatemala, Guiana, México (Chiapas), Panamá, Paraguai, Venezuela. N. youngheae Kim, 1972, sinônima de N. flavicoxa, tem como localidade-tipo Mato Grosso do Sul, Maracaju.

\section{Lista de espécies de Ulidiidae no Estado do Mato Grosso} do Sul

\section{Notogramma Loew, 1868}

cimiciforme Loew, 1868. Localidade-tipo: Cuba. Distribuição Neotropical: Brasil, Colômbia, Cuba, Equador, Guiana, Jamaica, México ao Panamá, Peru, Venezuela.

Agradecimentos. Os autores agradecem a Fundação de Apoio ao Desenvolvimento do Ensino, Ciências e Tecnologia do Estado de Mato Grosso do Sul (Fundect) e a Superintendência de Ciências e Tecnologia do Estado de Mato Grosso do Sul (Sucitec/MS) pelo convite de participação neste fascículo especial da Iheringia, Série Zoologia e o suporte financeiro para sua publicação; e as seguintes agências de fomento à pesquisa: Conselho Nacional de Desenvolvimento Científico e Tecnológico (CNPq); Fundação de Amparo à Pesquisa do Estado de São Paulo (FAPESP); Fundação de Apoio à Pesquisa do Distrito Federal (FAPDF); Coordenação de Aperfeiçoamento de Pessoal de Nível Superior (CAPES); Financiadora de Estudos e Projetos (FINEP), Pró-Reitoria de Pesquisa e Pós-Graduação da Universidade Católica de Brasília (UCB).

\section{REFERÊNCIAS BIBLIOGRÁFICAS}

Aguiar-Menezes, E. L.; Nascimento, R. J. \& Menezes, E. B. 2004. Diversity of fly species (Diptera: Tephritoidea) from Passiflora spp. and their hymenopterous parasitoids in two municipalities of the Southeastern Brazil. Neotropical Entomology 33(1):113-116.

Allen, E. J. \& Foote, B. A. 1992. Biology and immature stages of Chaetopsis massyla (Diptera: Otitidae), a secondary invader of herbaceous stems of wetland monocots. Proceedings of the Entomological Society of Washington 94:320-328.

Barnabe, A. S.; Laporta, G. Z.; Laporta, M. Z. \& Lamas, C. J. E. 2007. New record of Pterotaenia fasciata (Wiedemann) (Diptera, Ulidiidae) in Brazil, a probably mechanical vector of enteric bacteria. Revista Brasileira de Entomologia 51(1):121-122.

Barnes, J. K.; Slay, M. E. \& TAYLOR, S. J. 2009. Adult Diptera from Ozark Caves. Proceedings of the Entomological Society of Washington 111(2):335-353.

Belshaw, R. 1989. A note on the recovery of Thoracochaeta zosterae (Haliday) (Diptera: Sphaeroceridae) from archaeological deposits. Circaea 6(1):39-41.

BERgERON, M. D. 2010. A world review of Coproica Rondani (Diptera; Sphaeroceridae) with a revision of the new world species. Dissertação de mestrado. Guelph, University of Guelph.

Bertolaccini, I.; Bouzo, C.; Larsen, N. \& Favaro, J. C. 2010. Especies del género Euxesta (Diptera: Ulidiidae = Otitidae) plagas de maíces dulces Bt en la provincia de Santa Fe, Argentina. Revista de la Sociedad Entomológica Argentina 69(1-2):123-126.

Bjerke, J. M.; Anderson, A. W. \& Freeman, T. P. 1992. Morphology of the larval stages of Tetanops myopaeformis (Röder) (Diptera: Otitidae). The Journal of the Kansas Entomological Society 65(1):59-65.

Boff, S.; Graciolli, G.; Boareto, A. G. \& Marques, M. R. 2008. Insetos visitantes de gomas exsudadas por Terminalia argentea Mart \& Zucc (Combretaceae). Revista Brasileira de Entomologia 52(3):477-479.

BRUnel, O. \& Rull, J. 2010. Natural history and mating behavior of Pseudodyscrasis scutellaris, a fly species (Ulidiidae) associated with agave in Mexico. Annals of the Entomological Society of America 103(3):430-438.

Buck, M. 1997. Sphaeroceridae (Diptera) reared from various types of carrion and other decaying substrates in Southern Germany, including new faunistic data on some rarely collected species. European Journal of Entomology 94:137-151.

Buck, M. \& Marshall, S. A. 2009. Revision of New World Leptocera Olivier (Diptera, Sphaeroceridae). Zootaxa 2039:1-139.

Buxton, P. A. 1960. British Diptera associated with fungi. Flies of all families reared from about 150 species of fungi. Entomologist's Monthly Magazine 96:61-94. 
CoRnaby, B. W. 1974. Carrion reduction by animals in contrasting tropical habitats. Biotropica 6(1):51-63.

Cruz, I.; Silva, R. B.; Figueredo, M. de L. C.; Penteado-Dias, A. M.; SARTO, M. C. L. Del \& Nuessly, G. S. 2011. Survey of ear flies (Diptera, Ulidiidae) in maize (Zea mays L.) and a new record of Euxesta mazorca Steyskal in Brazil. Revista Brasileira de Entomologia 55(1):102-108.

DiAz, W. 1982. Daños de Euxesta eluta y E. mazorca (Dipt.: Otitidae) sobre maíces amiláceos em la Costa Central del Perú. Revista Peruana de Entomología 25(1):51-53.

FISCHER, C. R. 1932. Um gênero e duas espécies novas de Rhopalomeridae do Brasil, e o pupário de Willistoniella pleuropunctata Wied. (Dipt.). Revista de Entomologia 2:441-450.

Foote, R. H. 1960. The Tephritidae and Otitidae of the Bahama Islands (Diptera). Journal of the New York Entomological Society 68(2):8399.

Gómez, L. D. 1977. La mosca del cangrejo terrestre Cardisoma crassum Smith (Crustacea: Gecarcinidae) en la Isla del Coco, Costa Rica. Revista de Biologia Tropical 25(1):59-63.

Goyal, G.; Nuessly, G. S.; Steck, G. J.; Dakshinar, S. R.; Capinera, J. L. \& Boоte, A. J. 2010. New report of Chaetopsis massyla (Diptera: Ulidiidae) as a primary pest of corn in Florida. Florida Entomologist 93(2):198-202.

IbÁÑez-Bernal, S. \& Hernández-Ortiz, V. 2010. Ropalomeridae (Ropalomeridae Flies). In: Brown, B. V.; Borkent, A.; Cumming, J. M.; Wood, D. M.; Woodley, N. E. \& Zumbado, M. eds. Manual of Central American Diptera. Ottawa, NRC Research Press. vol. 2, p.1025-1030.

Kameneva, E. P. 2004a. New records of picture-winged flies (Diptera: Ulidiidae) of Central America. Studia Dipterologica 10:609-652.

Kameneva, E. P. 2004b. A new species of the genus Plagiocephalus (Diptera, Ulidiidae) from Central America. Vestnik Zoologii 38:15-22.

Kameneva, E. P. 2005. A new genus and species of the tribe Lipsanini (Diptera, Ulidiidae) from Central America. Vestnik Zoologii 39:99-103.

Kameneva, E. P. 2009. A new species of the genus Cymatosus (Diptera, Ulidiidae) from Central America, with a key to species. Vestnik Zoologii 43:267-273.

Kameneva, E. P. \& Korneyev, V. A. 2006. Myennidini, a new tribe of the subfamily Otitinae (Diptera: Ulidiidae), with discussion of the suprageneric classification of the family. Israel Journal of Entomology 35-36:497-586

Kameneva, E. P. \& Korneyev, V. A. 2010. Ulidiidae (Pictured-winged flies). In: Brown, B. V.; Borkent, A.; Cumming, J. M.; Wood, D. M.; Woodley, N. E. \& Zumbado, M. eds. Manual of Central America Diptera. Boca Raton, NRC Research Press. vol. 2, p.883-904.

KIM, K. C. 1972. The New World genus Parasphaerocera and allied groups, with descriptions of new genera and species (Diptera: Sphaeroceridae). Miscellaneous Publications of the Entomological Society of America 8(6):377-444.

Lopes, H. S. DE 1932. Sobre a Rhopalomera stictica Wied. 1828. (Dipt. Rhopalomeridae). Anais da Academia Brasileira de Ciências 4:127129.

Manrique-Saide, P. C. \& Delfín-González, H. 1997. Importancia de las moscas como vectores potenciales de enfermedades diarréicas em humanos. Revista Biomédica 8:163-170.

Marchiori, C. H. \& Silva, C. G. 2000. Dípteros Sinantrópicos Associados a Restos Alimentares e Seus Parasitóides. Neotropical Entomology 30(1):187-189.

Marques, A. P. C. \& Ale-Rocha, R. 2005. Revisão do gênero Willistoniella Mik, 1895 (Diptera, Ropalomeridae) da Região Neotropical. Revista Brasileira de Entomologia 49:210-227.

Marshall, S. A. 1995. Sclerocoelus and Druciatus, new genera of New World Sphaeroceridae (Diptera; Sphaeroceridae; Limosininae). Insecta Mundi 9:283-289.

Marshall, S. A. 1997a. A revision of the Sclerocoelus galapagensis group (Diptera: Sphaeroceridae: Limosininae). Insecta Mundi 11:97-115.

Marshall, S. A. 1997b. A first record of Aptilotus Mik (Diptera: Sphaeroceridae) from the Neotropical Region, with the description of three new wingless species of the Aptilotus paradoxus group from high elevations in Costa Rica. Proceedings of the Entomological Society of Washington 99:505-512.
Marshall, S. A. 2001. A review of the southern South American genus Gyretria Enderlein (Diptera: Sphaeroceridae: Limosininae). Proceedings of the Entomological Society of Washington 103:282290.

Marshall, S. A. \& Buck, M. 2010. Sphaeroceridae (small dung flies). In: Brown, B. V.; Borkent, A.; Cumming J. M.; Wood D. M.; Woodley, N. E. \& Zumbado, M. A. eds. Manual of Central America Diptera. Ottawa, NRC Research Press. vol. 2, 1165-1187.

Marshall, S. A.; Buck, M. \& Lonsdale, O. 2007: Lepidosina, a new genus of New World Limosininae (Diptera: Sphaeroceridae). European Journal of Entomology 104:573-599.

Marshall, S. A. \& Norrbom, A. L. 1992. A revision of the New World species of Norrbomia (Diptera: Sphaeroceridae), including all American species previously placed in Borborillus. Insecta Mundi 6(3-4):151181.

Marshall, S. A. \& Richards, O. W. 1987. Sphaeroceridae. In: MCAlpine, J. F.; Peterson, B. V.; Shewell, G. E.; Teskey, H. J.; Vockeroth, J. R. \& Wood, D. M. eds. Manual of Nearctic Diptera. Ottawa, Agriculture Canada Monograph. vol. 2, p. 993-1006.

Marshall, S. A.; RoháčeK, J.; Dong, H. \& BuCK, M. 2011. The state of Sphaeroceridae (Diptera: Acalyptratae): a world catalog update covering the years 2000-2010, with new generic synonymy, new combinations, and new distributions. Acta Entomologica Musei Nationalis Pragae 51(1):217-298.

McAlpine, J. F. 1981. Morphology and terminology - Adults. In: MCAlPINE, J. F.; Peterson, B. V.; Shewell, G. E.; Teskey, H. J.; Vockeroth, J. R. \& Wood, D. M. eds. Manual of Neartic Diptera. Ottawa, Research Branch Agriculture Canada. vol. 1, p. 9-63.

Mendes, J. \& Linhares, A. X. 2002. Cattle dung breeding Diptera in pastures in southeastern Brazil: Diversity, abundance and seasonallity. Memórias do Instituto Oswaldo Cruz 97(1):37-41.

Micks, D. W. \& McKibBEn, J. W. 1956. Report on case of human intestinal myiasis caused by Leptocera venalicia. The American Journal of Tropical Medicine and Hygiene 5:929-932.

Mourgués-Schurter, L. R. 1981. Sobre as espécies neotropicais de Neosphaerocera Kim, 1972 (Diptera, Sphaeroceridae). Papéis Avulsos de Zoologia 34(18):179-188.

Niogret, J. \& Lumaret, J. P. 2009. Identification of the cues used in the host finding behavior during the phoretic association Ceroptera rufitarsis (Diptera: Sphaeroceridae) and dung beetles (Coleoptera: Scarabaeidae). Journal of Insect Behavior 22:464-472.

Norrbom, A. L. \& KIM, K. C. 1984. Taxonomic status of Lotophila Lioy, with a review of $L$. atra (Meigen) (Diptera: Sphaeroceridae). Proceedings of the Entomological Society of Washington 86(2):305-311.

Pape, T.; Blagoderov, V. \& Mostovski, M. B. 2011. Animal Biodiversity - Order Diptera. Zootaxa 3148:222-229.

Prado, A. P. 1963. Primeira contribuição ao conhecimento da família Rhopalomeridae (Diptera). Memórias do Instituto Oswaldo Cruz 61:459-470.

Prado, A. P. 1966. Segunda contribuição ao conhecimento da família Rhopalomeridae (Diptera, Acalyptratae). Studia Entomologica 8(1965):209-268.

Ribeiro, M. F.; Köhler, A. \& Boelter, C. R. 2006. Polinizacão de Acianthera aphtosa (lindl.) Pridgeon \& M. W. Chase (Orchidaceae) por Otitidae (Diptera). Revista da FZVA 13(2):85-89.

RicHARDS, O. W. 1965. A contribution to the study of the genus Sphaerocera Latreille in Central and South America (Diptera: Sphaeroceridae). Proceedings of the United States National Museum 116:223-242.

RICHARDS, O. W. 1967. Family Sphaeroceridae (Borboridae) In: PAPAVERO, N. ed. A catalogue of the Diptera of the Americas South of the United States. São Paulo, Departamento de Zoologia, Secretaria de Agricultura. vol. 72, p.1-28.

Rodrigues, S. R.; Nantes, L. R; Souza, S. R. DE; Abot, A. R. \& UchôAFernandes, M. A. 2006. Moscas frugívoras (Diptera, Tephritoidea) coletadas em Aquidauana, MS. Revista Brasileira de Entomologia 50(1):131-134.

RoHÁČEK, J. \& MARSHALl, S. A. 1988. A review of Minilimosina (Svarciella) Rohácek, with descriptions of fourteen new species (Diptera: Sphaeroceridae). Insecta Mundi 2:241-282.

RoháčeK, J. \& MARShall, S. A. 1998. Revision of Homalomitrinae subfam.n. (Diptera: Sphaeroceridae), with the description of a new genus and three new species. European Journal of Entomology 95:455-491. 
RoháČEK, J.; Marshall, S. A.; Norrbom, A. L.; Buck, M.; Quiros, D. I. \& SMITH, I. 2001. In: RoHÁČEK, J. ed. World catalog of Sphaeroceridae. Opava, Slezské Zemské Muzeum. 300p.

Smith, I. P. \& Marshall, S. A. 2004: A review of the New World Genus Pterogramma Spuler and a revision of the Pterogramma sublugubrinum group. Contributions in Science 499:1-163.

SouzA, A. M. \& Linhares, A. X. 1997. Diptera and Coleoptera of potential forensic importance in southeastern Brazil: relative abundance and seasonality. Medical and Veterinary Entomology 11:8-12.

Steyskal, G. C. 1957. The postabdome of male Acalyptratae Diptera. Annals of the Entomological Society of America 50:66-73.

Steyskal, G. C. 1967. Family Ropalomeridae. In: Vanzolini, P. E. \& PAPAVEro, N. eds. A catalogue of the Diptera of the Americas South of the United States. Departamento de Zoologia, Secretaria de Agricultura, São Paulo. vol. 60, p.1-7.

SteysKal, G. C. 1968. Family Otitidae (Ortalidae; including Pterocallidae,
Ulidiidae). In: Papavero, N. ed. A catalogue of the Diptera of the Americas South of the United States. São Paulo, Departamento de Zoologia, Secretaria de Agricultura. vol. 54, p.1-23.

Steyskal, G. C. 1971. A new central american species of Zacompsia Coquillett, with a key to the described species (Diptera: Otitidae). Proceedings of the Entomological Society of Washington 73:247-248.

Teskey, H. J. 1976. Diptera larvae associated with trees in North America. Memoirs of the Entomological Society of Canada 108:1-53

UchôA-Fernandes, M. A.; Oliveira, I. DE; Molina, R. M. S. \& Zucchi, R. A. 2002. Species diversity of frugivorous flies (Diptera: Tephritoidea) from hosts in the Cerrado of the state of Mato Grosso do Sul, Brazil. Neotropical Entomology 31(4):515-524.

UchôA-Fernandes, M. A.; Oliveira, I. De; Molina, R. M. S. \& Zucchi, R. A. 2003. Biodiversity of frugivorous flies (Diptera: Tephritoidea) captured in citrus groves, Mato Grosso do Sul, Brazil. Neotropical Entomology 32(2):239-246 